\title{
CONSTRUCTION AND OPERATION OF HIGH POWER GAS LASERS
}

\author{
W.J. WITTEMAN, G.J. ERNST and P.J.M. PETERS
}

University of Twenty, Enschede, The Netherlands

In the present paper some typical gas laser construction and their performances will be described. Aspects of transition selective systems and high pressure operation will be treated.

\section{High quality transition selective molecular systems}

The usual construction for transition selection is a cavity with a curved mirror on one side and a tunable grating in the Littrow position at the other side. The reflection losses at the grating can be considerable, especially if the tuned frequency is not at the blazed angle. For some molecular systems like a room temperature $\mathrm{CO}$ laser where the gain is low the quality of cavity may become too low and a great deal of the stimulated emission will be lost if laser action will occur at all. A considerable improvement of the reflection, seen by the incident radiation on the grating, is obtained by placing a supporting reflector in front of the grating as shown in fig. 1 [1]. If this mirror is perpendicular to the optical axis it forms with the grating a Fabry-Pérot (FP). The incident radiation on this FP experiences a complex amplitude reflection given by:

$\sqrt{R_{\mathrm{tot}}} \mathrm{e}^{\mathrm{i} \phi}=-\sqrt{R_{1}}+\frac{\left(1-R_{1}\right) \sqrt{R_{21}}}{\mathrm{e}^{-2 \mathrm{i} \gamma}-\sqrt{R_{1} R_{21}}}$

where $R_{1}$ is the intensity reflectivity of the supporting mirror and $R_{21}$ is the first-order intensity reflectivity of the grating; $\gamma=\omega L_{2} / c$ is the optical phase of the FP. It is seen that by changing the optical path $L_{2}$ the reflectivity can be much higher than that of the grating alone. For instance if the supporting mirror
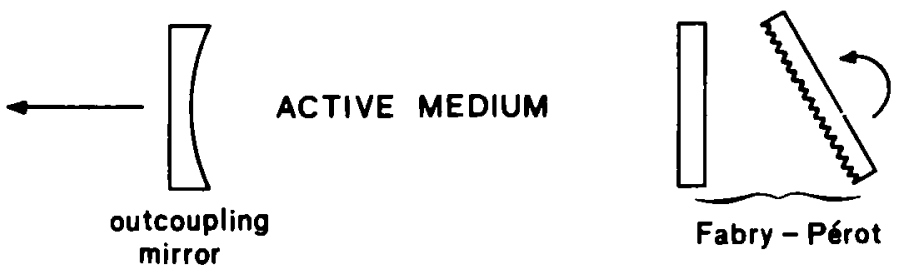

Fig. 1. A high quality frequency selective laser cavity. One end mirror is formed by a Fabry-Pérot containing a grating in the Littrow position. 
has an intensity reflectivity of $81 \%$ and the grating $64 \%$ the maximum of $R_{\text {tot }}$ becomes $97.5 \%$. Further the effect of an additional mirror is a considerable decrease of the power load at the grating by tuning at maximum reflectivity [1]. At high power the cooling of the grating is much less complicated.

The requirements for using such a transition selective device as a practical instrument are:

a. a stable three mirror configuration that after alignment will hold its position for a long period of time even after transportation.

b. automatic and stable adjustment of the FP for maximum reflectivity during operation.

These requirements can be fullfilled and are described in the following.

\subsection{STABLE THREE MIRROR CONFIGURATION}

The requirements mentioned above can be fulfilled with the construction shown in fig. 2 [2]. Inside a pyrex support tube the quartz discharge tube has been mounted. Both the pyrex support tube and the quartz laser tube are on one side glued in the central mounting block. In this solid block also the grating is mounted with the possibility to turn it very precisely around a vertical axis perpendicular to the optical axis. We used a 18001 /inch gold-coated grating, blazed at $10.6 \mu \mathrm{m}$. Further the grating is mounted on a piezo-electric translator, in order to tune the length of the FP at the oscillating wave-length. The other side of the quartz discharge tube near the outcoupling mirror is connected to the pyrex support tube by means of a slightly adjustable support mechanism. It should be noticed that the pyrex support tube is fixed only on one side to the central mounting block. This block, on its turn, is mounted to a thick, rigid, iron profile. The laser tube has a discharge length of about $90 \mathrm{~cm}$ and an internal diameter of $9 \mathrm{~mm}$. The electrode material is gold. Near the grating a flat mirror

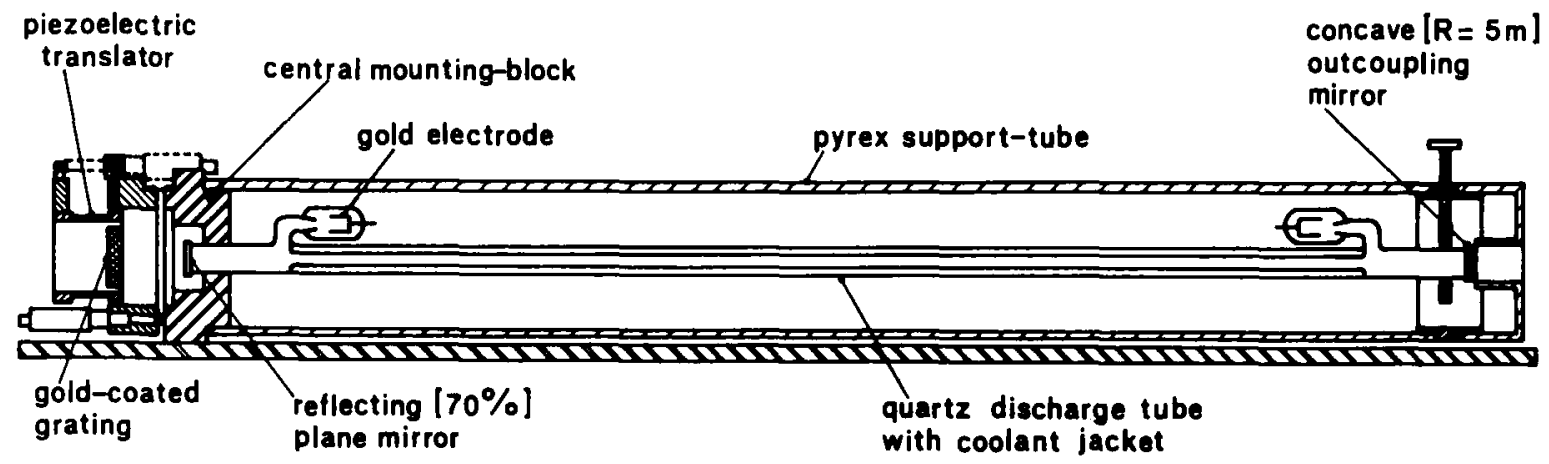

Fig. 2. Technical drawing of the cross section of a stable tunable $\mathrm{CO}_{2}$ laser. Note that the pyrex support-tube is only on one side supported. 
with $80 \%$ reflectivity is used. The outcoupling mirror has a curvature of $2 \mathrm{~m}$ and a reflectivity of $80 \%$. The stabilization of this device on a single line at the maximum reflectivity of the FP can be accomplished by means of the opto-voltaic effect as will be described in the following section.

\subsection{FREQUENCY AND OUTPUT STABILIZATION BY THE OPTO-VOLTAIC EFFECT}

The phenomenon of static resistance fluctuation in a cw laser discharge due to the intra cavity coherent radiation is well-known as opto-voltaic or opto-galvanic effect [3]. It can be used successfully for stabilizing a single line $\mathrm{CO}_{2}$ laser with high accuracy [3-5], the alignment of a laser cavity [6], and the detection of radiation [7] in $\mathrm{CO}_{2}$ lasers. In general the resistance fluctuations of the discharge due to stimulated emission in a current stabilized or ballast resistor regime result in fluctuations of the power that is dissipated in the discharge, and hence in temperature variations of the plasma and the discharge tube. The relative changes of static resistance of the laser tube due to changes of stimulated emission inside the laser cavity can be expressed in terms of relative changes of the tube voltage $U_{\mathrm{L}}$ (or longitudinal electric field) and the discharge current $I$ :

$$
\frac{\Delta R_{\mathrm{S}}}{R_{\mathrm{S}}}=\frac{\Delta U_{\mathrm{L}}}{U_{\mathrm{L}}}-\frac{\Delta I}{I}
$$

We shall consider the discharge laser tube as a nonlinear resistance with parametrical change of its voltage-current characteristic by the coherent radiation in the discharge tube. The operating current and voltage on the characteristic are determined by the voltage, $E_{\mathrm{a}}$, of the power supply and the serial balast resistor, $R_{\mathrm{b}}$, the value of which must be larger than the absolute value of the negative dynamic resistance of discharge tube (fig. 3). The operating values of $U_{\mathrm{LO}}$ and $I_{\mathrm{O}}$ changes with the radiation power along the working-line determined by balast resistor $R_{\mathrm{b}}$ and supply voltage $E_{\mathrm{a}}$ :

$$
U_{\mathrm{LO}}=E_{\mathrm{a}}-R_{\mathrm{b}} I_{\mathrm{O}}
$$

It is seen from fig. 3 that the displacement along this line involves the change of discharge voltage, $\Delta U_{\mathrm{L}}$, and simultaneously the change of discharge current, $\Delta I$. The impedance of the discharge tube, however, increases with the produced radiation. This means that the variation of the discharge voltage, $\Delta U_{\mathrm{L}}$, called opto-voltaic effect is in phase with the variation of the radiation power $P$, and simultaneously the variation $\Delta I$, called opto-galvanic effect, is in opposite phase. The opto-galvanic signal is usually measured over a probe resistor connected in series between cathode and earth. A first order approximation of the static 


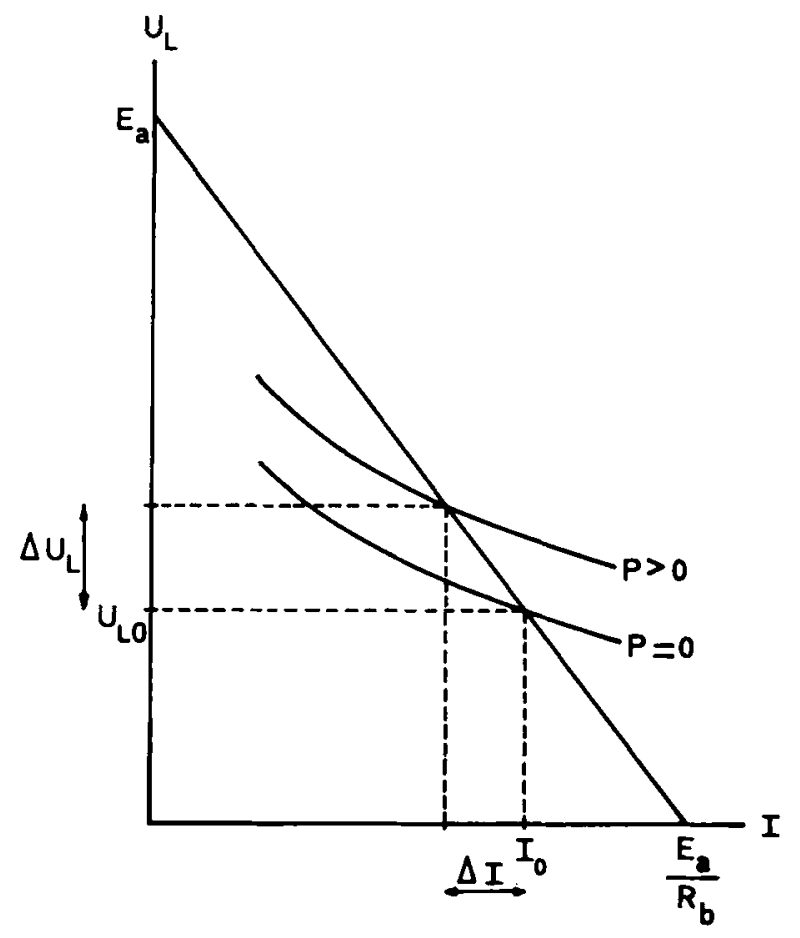

Fig. 3. Illustration of voltage-current characteristic of a laser tube parametrically changed by the power of radiation inside the cavity.

current-voltage characteristic of the discharge can be obtained by an expansion in power series in the neighbourhood of the point: $I=I_{\mathrm{O}}, P=0$. We obtain:

$U_{\mathrm{L}}(I, P)=U_{\mathrm{L}}\left(I_{\mathrm{O}}, P=0\right)+\frac{\partial U_{\mathrm{L}}(I, P)}{\partial I}\left(I-I_{\mathrm{O}}\right)+\frac{\partial U_{\mathrm{L}}(I, P)}{\partial P} P$

or

$U_{\mathrm{L}}(I, P)=E_{\mathrm{b}}-R_{\mathrm{d}} I+\alpha P$

where:

$R_{\mathrm{d}}=-\frac{\partial U_{\mathrm{L}}}{\partial I}$ for $P=0$ and $I=I_{\mathrm{O}}$ is the dynamic resistance,

$E_{\mathrm{b}}=U_{\mathrm{LO}}+R_{\mathrm{d}} I_{\mathrm{O}}$

$\alpha=\frac{\partial U_{\mathrm{L}}}{\partial P}$ for $P=0$ and $I=I_{\mathrm{O}}$. 


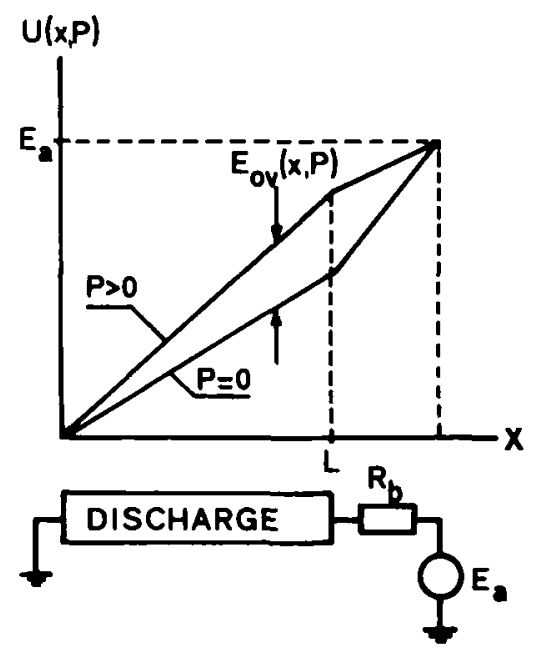

Fig. 4. Voltage drop $U_{\mathrm{L}}(x, P)$ along the laser tube for a laser with current stabilizer.

The opto-galvanic and opto-voltaic signals can be found from (3) and (5) as function of power:

$\Delta U_{\mathrm{L}}=\frac{\alpha R_{\mathrm{b}} \Delta P}{R_{\mathrm{b}}-R_{\mathrm{d}}}$

$\Delta I=\frac{\alpha \Delta P}{R_{\mathrm{d}}-R_{\mathrm{b}}}$.

The relative change of the impedance becomes:

$$
\frac{\Delta R}{R}=\left(\frac{\alpha R_{\mathrm{b}}}{E_{\mathrm{b}} R_{\mathrm{b}}-E_{\mathrm{a}} R_{\mathrm{d}}}+\frac{\alpha}{E_{\mathrm{a}}-E_{\mathrm{b}}}\right) \Delta P .
$$

In the case of current stabilization only the opto-voltaic effect is present.

It is interesting to make an analysis of voltage drop $U(x)$ along the laser tube. Figure 4 shows the voltage drop in the case of a current stabilizer. It is seen that the largest opto-voltaic effect is obtained near the ballast resistor $R_{\mathrm{b}}$ of the laser tube. The signal $E_{\mathrm{ov}}(x, P)$ is the modulated part of the plasma potential and it is linearly distributed along the tube. The total ballast resistance is $R_{\mathrm{b}}+R_{\mathrm{s}}$, where $R_{\mathrm{s}}$ is the dynamic resistance of the stabilizer which is much larger than $R_{\mathrm{d}}$. With this condition (6) becomes:

$\Delta U_{\mathrm{L}}=\alpha \Delta P$ and thus:

$$
E_{\mathrm{ov}}(x, P)=\alpha P \frac{(L-x)}{L} .
$$


The extra power $Q$ that is delivered to the discharge, the so called optovoltaic input due to laser action is then given by:

$Q=E_{\mathrm{ov}}(x=0, P) \cdot I$,

For a molecular laser like $\mathrm{CO}$ and $\mathrm{CO}_{2}$ the discharge tube is surrounded by a water jacket. In this way the plasma is capacitively coupled to the water jacket. The initial linear distribution of the polarization charges due to $E_{\mathrm{ov}}(x, P)$ will equilibrate over the water jacket because of its conductivity. For an usual quartz tube laser construction the capacity over the quartz wall can be estimated as 6 $\mathrm{pF} / \mathrm{cm}$ and the resistance through the cooling water as $1.5 \mathrm{k} \Omega / \mathrm{cm}$. For a one meter tube the corresponding equilibrium time $\tau$ is about $9 \cdot 10^{-5} \mathrm{sec}$. With this time constant the potential of the water jacket reaches a maximum amplitude $\tilde{E}_{\text {ov }}=\frac{1}{2} E_{\text {ov }}(x=0, P)$. This value can experimentally be observed and turns out to be approximately in a range of typical modulation frequencies up to $1 \mathrm{kHz}$.

\subsection{OPTO-VOLTAIC SIGNAL IN THE WATER COOLING-JACKET}

To demonstrate the opto-voltaic signal we chop the radiation within the cavity. We detect the opto-voltaic signal in the water-jacket by putting a needle-shaped probe in the water tube close to the water-jacket [5]. The signal $S_{2}$ in fig. 5, measured close to the water-jacket, is the response to the nearly rectangular opto-voltaic signal in the differential circuit $R C_{1}$, where $R$ is the resistance of about $1 \mathrm{M} \Omega$ of the scope and $C_{1}$ is the capacity between the discharge and the water-jacket. For the reproduction of the nearly rectangular shape of the opto-voltaic signal we use a capacitor $C_{2}=2.2 \mu \mathrm{F}$ in series with the needle. In this way we effectively construct a capacitive voltage divider (because the resistance of the water between the needle-probe and earth was much higher than the reactance of the $C_{2}$ capacitor) consisting of the capacity $C_{1}$ between the plasma and the water jacket and $C_{2}$ between the water jacket and earth.

In fig. 5 we plot the signals $S_{1}$ measured on the electrode $(x=0), S_{2}$ measured on the needle with a resistance of $1 \mathrm{M} \Omega$, and $S_{3}$ measured with the voltage divider between $C_{1}$ and $C_{2}$. We observed that $S_{1}$ and $S_{3}$ have the same shape and the relative amplitudes are in agreement with the ratio of the capacitive divider.

Usually opto-voltaic detection is done by means of a high impedance probe on the cathode or anode depending on the supply regime. Here we show that the opto-voltaic signal can also be obtained capacitively from the water jacket with a simple needle probe. The advantage of this method is its high sensitivity and the separation from the high voltage circuit. Because of its sensitivity it can be successfully used for frequency stabilization of the three mirror system. Applying 


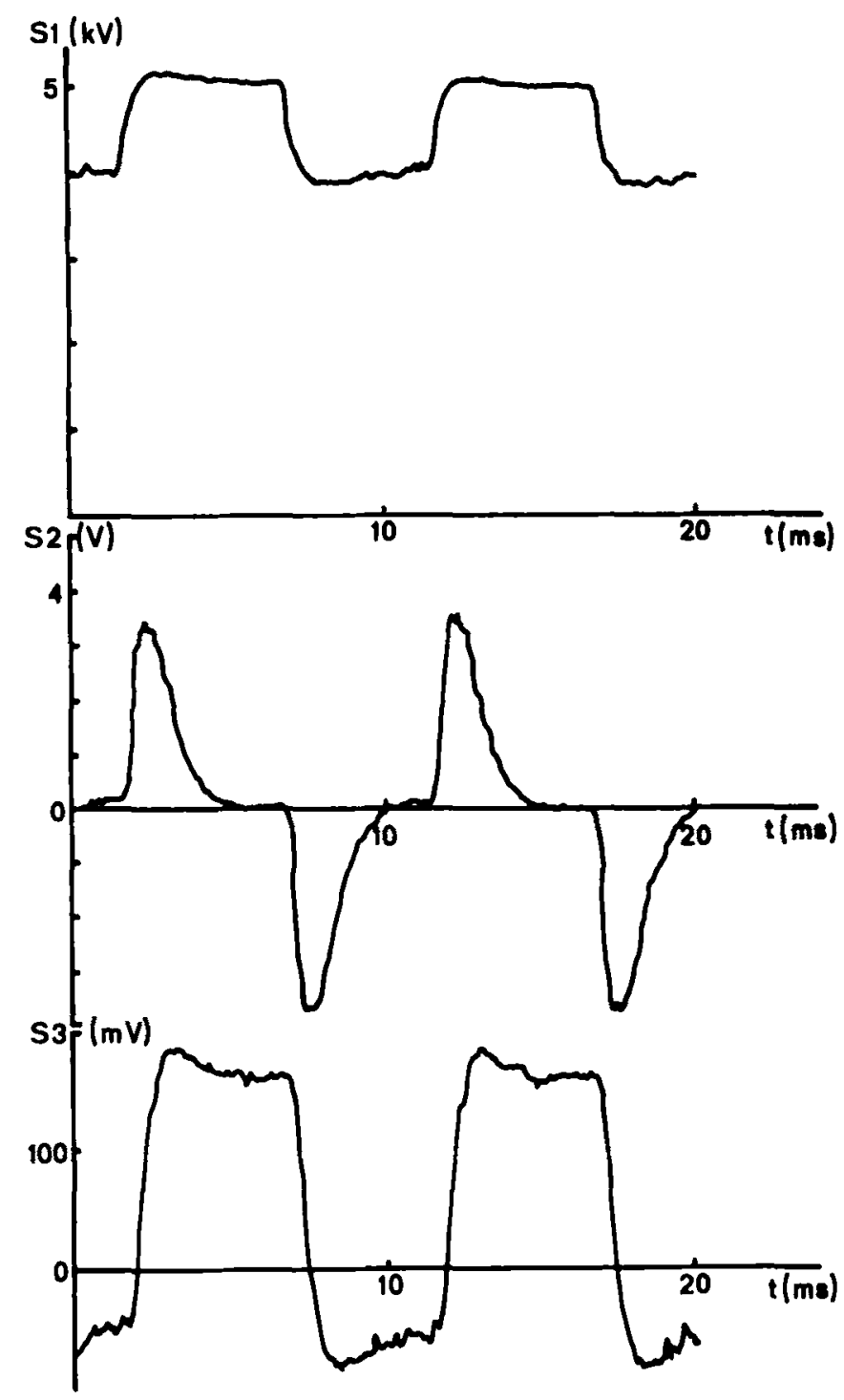

Fig. 5. Oscillograms of the opto-voltaic signals due to intracavity chopping obtained at the electrodes $\left(S_{1}\right)$, directly on the needle $\left(S_{2}\right)$, and by means of a voltage divider with a capacity of $2.2 \mu \mathrm{F}$ in series with the needle $\left(S_{3}\right)$.

a small oscillating voltage to the piezoelectric translator of the construction shown in fig. 2 the capacitively coupled voltage signal $\left(S_{3}\right)$ of the water jacket is phase sensitively detected and used to optimize the output power by means of a d.c. voltage component applied to the piezo-electric element. In other words the radiation frequency is stabilized near the line center frequency. It is observed that 
the minimum amplitude of this translator is about one nanometer which corresponds to a frequency variation of about $40 \mathrm{kHz}$.

\section{Pulsed systems with dielectric corona preionization}

The preionization in a pulsed discharge provides the initial electrons in the main electrode gap that condition the start of a glow discharge between the electrodes. Experiments have indicated that uniform preionization is highly desired. For this it is very important that the UV source is uniform and extends over an area equal to the length of the electrodes times their separation distance. The most successful technique of reaching this is the use of a dielectric corona discharge. The term corona discharge often refers to a phenomenon which occurs in a gaseous medium in the vicinity of conductors of small radius of curvature, subjected to high voltages. The high electric field along the surface which usually is non-uniform, produces a partial-breakdown of the surrounding gas. A similar situation may arise when a dielectric plate is subjected to a high electric field. The field polarizes the dielectric plate and a high field is present at its surface. A corona discharge may then be produced in the surrounding gas near the dielectric.

The physical principles involved in producing a uniform preionization in a laser is explained with the aid of fig. 6. This figure shows a cross section of the laser cavity perpendicular to the optical axis. The contours of anode and cathode are according to an uniform electric field distribution at their surfaces. Through an electric circuit a high voltage pulse is suddently applied between the electrodes. Thus a high electric field with a rapid rise appears between them. Because

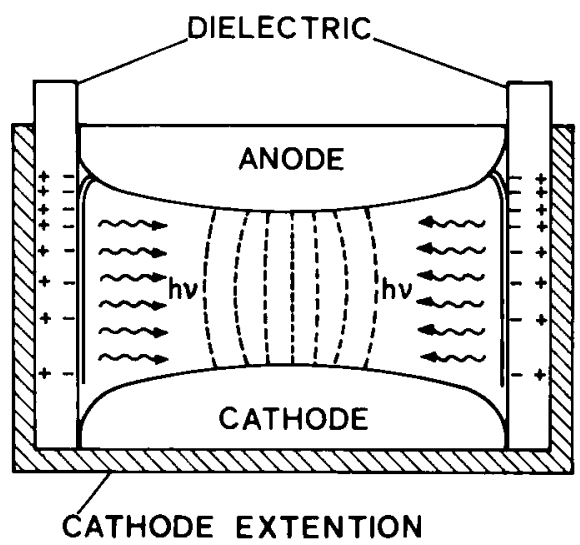

Fig. 6. Schematic drawing of the corona discharge along the dielectric material and the UV-radiation that preionizes the main discharge. 
the cathode has a metallic extension bent around the two dielectric plates reaching closely the anode the dielectric plates are subjected to a very strongelectric field. They become polarized with surface charges as shown in fig. 6 . The high voltage at the surrounding gas produces a surface corona discharge. The formed ions in the surrounding gas are attracted by the dielectric plates and the electrons are easily pulled from the gas layer by the strong electric field in the corner formed by the dielectric and the anode. Once sufficient electrons are present in the gas layer streamers are formed which rapidly develop into avalanches. Because of the non-conductivity of the dielectric the surface discharge is uniform in the direction of the optical axis; i.e perpendicular to the figure. The produced plasma near the dielectric plates forms a channel with a width equal to the length of the electrodes for the passage of current from the anode to the cathode.

\subsection{SINGLE DISCHARGE CORONA PREIONIZED SYSTEMS}

In the previous section we described the performance of preionization by means of a dielectric surface in the surrounding laser gas. Realizations of the basic idea of a rapid corona discharge in a self sustained TEA laser are described in literature [8-10]. It has been shown that this type of self-sustained TEA lasers is superior to other types. This is because of its uniform preionization, its potential of relatively large electrode gap, and the simplicity of the single discharge. It has been shown that the technique can be used even for an electrode gap of $10 \mathrm{~cm}$ [10]. According to the work of ERNST and BOER the output ranges from 60 Joule/liter for a $2 \mathrm{~cm}$ up till 40 Joule/liter for a $10 \mathrm{~cm}$ electrode gap. The lower output per volume for larger electrode gap is due to the UV absorption loss from the corona to the discharge area. In fact the high output energy at $10 \mathrm{~cm}$ electrode gap is obtained in a relatively poor $\mathrm{CO}_{2}$ mixture of $\mathrm{CO}_{2}: \mathrm{N}_{2}: \mathrm{He}=1: 1: 10$. The uniform preionization of this type of self-sustained discharge allows for high excitation rate, i.e. high input energy, comparable to those of UV or electron beam sustained systems. Also the maximum small signal gain is comparable.

It is essential that the construction consists of uniform field electrodes and a very rapid power source. A typical construction as shown in fig. 7A was originally described by Ernst [10]. The electrodes are fabricated according to a Chang profile [11] for an electrode gap of $10 \mathrm{~cm}$. A $k$-value of 0.015 with $v=\operatorname{arc}$ $\cos (-k)$ has been chosen. The length of the profiles is $60 \mathrm{~cm}$ and their width is $364 \mathrm{~mm}$ or $346 \mathrm{~mm}$. For outcoupling a plane-parallel uncoated germanium flat is used. The glass plates as shown in the figure are glued to the electrodes. It was experimentally found that the thickness of the glass plates depends on the width of the electrodes. The smaller the width the thicker the plates. If the glass plates 


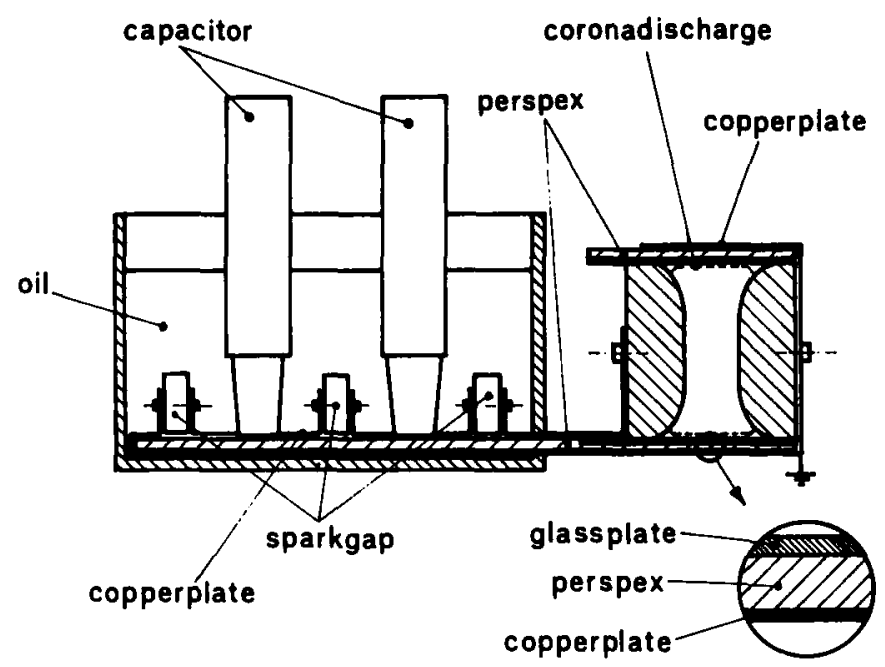

A

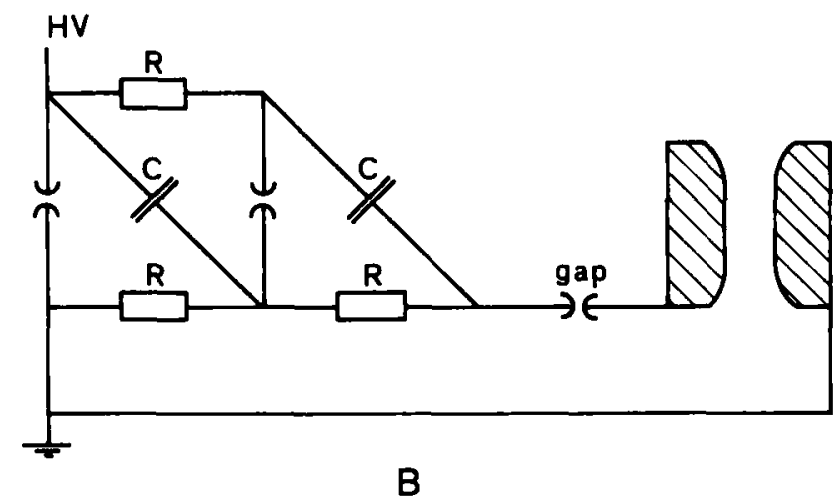

Fig. 7. a. Scheme of the construction of the system and details of the side-wall construction of the laser, b. Electrical scheme of the laser system.

are too thin $\mathrm{d} i / \mathrm{d} t$ of the corona discharge apparently degrades into a surface spark. Instead of changing the thickness the capacity for the displacement current can also be varied by placing a perspex plate between the glass plate and the copper plate. For the width of the electrodes of $364 \mathrm{~mm}$ the thickness of the glass plate and perspex plate is $13 \mathrm{~mm}$ and for the width of $346 \mathrm{~mm}$ it has an optimum of $23 \mathrm{~mm}$. The rapid discharge is obtained by using a Marx generator with low inductance. The electric scheme is shown in fig. 7B. It has two stages and an additional gap, which provides for the insulation of the laser head from the Marx generator when the first spark gap fires. For a sparking discharge the oscillation 


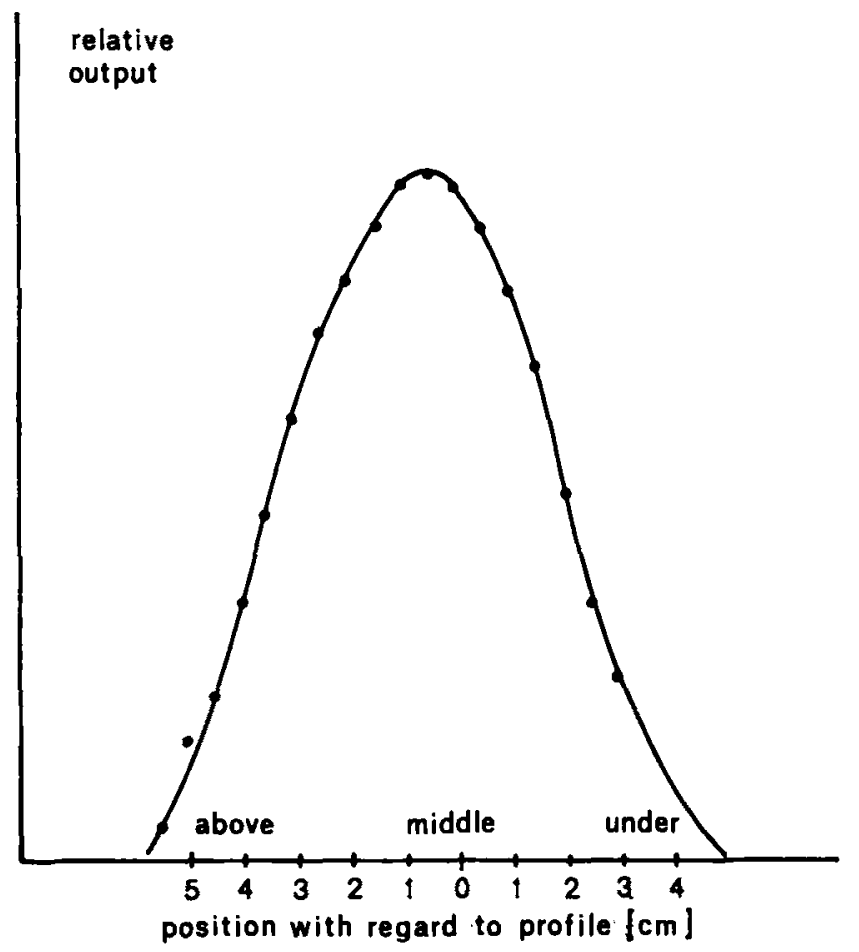

Fig. 8. Output energy density as a function of position between the electrodes for a $1: 1: 10$ mixture is shown. The capacity and Marx generator voltage are respectively $360 \mathrm{nF}$ and $45 \mathrm{kV}$ per stage [10].

frequency of the current through the system gives a rough indication of the self-inductance of the system. It was estimated as about $200 \mathrm{nH}$. The maximum output energy is 148 Joules (accuracy 10\%) for a Marx-generator voltage of $50 \mathrm{kV}$ per stage. The capacitance per stage of the Marx generator is $360 \mathrm{nF}$. Figure 8 shows the measured output beam diameter. The data are obtained by moving a 2 $\mathrm{mm}$ slit over the beam. It is observed that the beam is very uniform. The width of the ouput beam appears to be $55 \mathrm{~mm}$, which means an active volume of 3.3 liters. This results in an ouput energy of 40 Joules per liter. The efficiency calculated with an input energy of 729 Joules is $18.5 \%$.

\section{Table top e-beam pumped excimer lasers}

Rare-gas halogen exciplexes $\left(\mathrm{KrF}^{*}, \mathrm{XeF}^{*}, \mathrm{XeCl}^{*}\right.$, and $\left.\mathrm{ArF}^{*}\right)$ are used as active media to obtain the most powerful and efficient UV lasers to date. The exciplexes are produced in gas mixtures containing rare gases and a halogen gas. The excitation is achieved by irradiation of the gas mixture with a relativistic 
electron beam, or in an X-ray or UV preionized discharge. The best result with respect to homogeneity and efficiency of the laser beam is obtained by means of e-beam pumping. However the disadvantages of e-beam systems are their sizes, complexity, and costs. In this contribution we report on a table top e-beam system, which is relatively simple. It can be used for high gas densities, even above 10 bar, and simultaneous excitation current densities above $300 \mathrm{~A} / \mathrm{cm}^{2}$.

\subsection{DESCRIPTION OF THE E-BEAM SYSTEM}

We developed a high pressure coaxial system for high current density and a homogeneous excitation distribution.

The system shown in fig. 9 consists of a coaxial vacuum diode driven directly by a low-inductance ten-stage Marx generator [12,13]. The spark gaps are UV-triggered, except for the first gap, which is triggered externally. Each stage is formed by eight pairs of $\mathrm{BaTiO}_{3}$ capacitors of $1.8 \mathrm{nF}$ each. The total capacitance per stage is $7.2 \mathrm{nF}$. The UV-radiation of each firing gap can reach the next gap. In this way a fast, low jitter Marx generator is obtained.

The generator is enclosed in a cylindrical vessel of one meter height and 0.25 $\mathrm{m}$ diameter, filled with 4 bar $\mathrm{SF}_{6}$ to prevent flash-over. This compact arrangement provides a low inductance and a fast rise-time. The charging and ground-return resistors consist of two columns of silicon rubber tubing filled with coppersulphate solution; they are branched off at each stage. The resistance per stage is about $1 \mathrm{k} \Omega$, so that the internal discharge circuit of a firing gap has a time constant of about $7 \mu \mathrm{s}$. These resistors are capable of high power dissipation and are flexible enough to suit the electrical stressing requirements of the generator. The Marx generator is capable of producing 100 Joules at a maximum voltage of $600 \mathrm{kV}$. The spark gaps are flowed with dry air. The voltage is monitored by means of a differentiating voltage sensor and the current is recorded via a self-integrating Rogowski coil, both fitted near the diode as shown in fig. 9. Both signals can be displayed simultaneously on two transient digitizers. The cathode consists of a $20 \mathrm{~cm}$ long cylinder of $5 \mathrm{~cm}$ diameter, with rounded ends to prevent field emission or flash-over to the vacuum chamber walls. Inside this cylinder, two graphite felt strips of $20 \times 0.7 \mathrm{~cm}^{2}$ are mounted parallel to the axis, giving a two-sided transversal excitation in a radial symmetric electric field. The cylindrical anode laser tube was made of $50 \mu \mathrm{m}$ titanium foil with a laser weld along the axis. The diameter is $1 \mathrm{~cm}$ and the active volume is $16 \mathrm{~cm}^{3}$. It can be pressurized up to $15 \mathrm{~atm}$ under discharge conditions. Ti endpieces were also laser welded to this tube, after which a heat treatment at $700^{\circ} \mathrm{C}$ was used to remove internal strains. This ensured reliable operation of the anode tube. This cathode configuration gives a good impedance matching. The corresponding voltage and current waveforms are illustrated in figs. $10 \mathrm{a}$ and $\mathrm{b}$, respectively. We notice that 


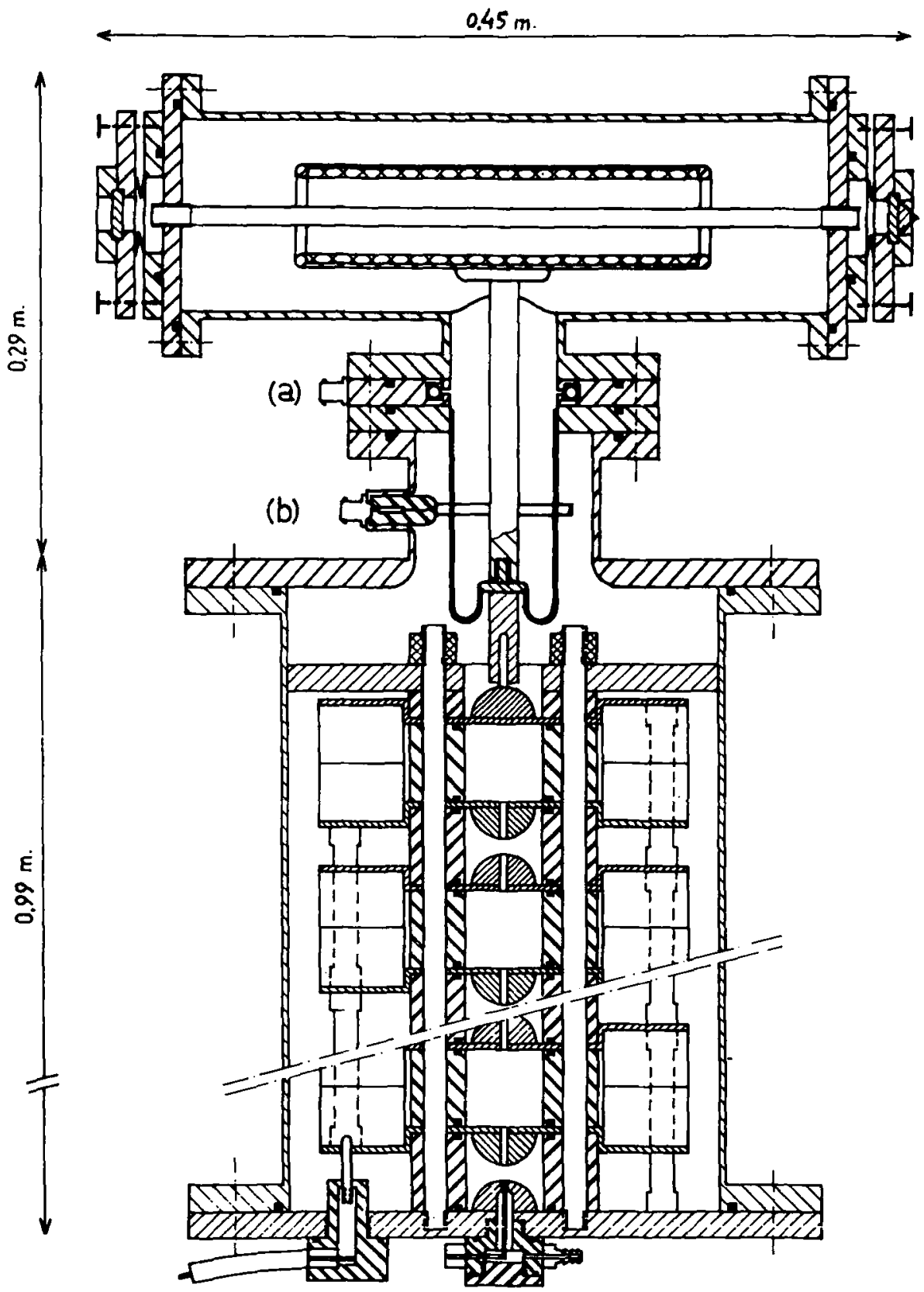

Fig. 9. Cross section of the e-beam pumped laser construction with a fast Marx generator (a): Rogowski coil for current measurements, (b): Capacitive coupled differentiating voltage sensor. 

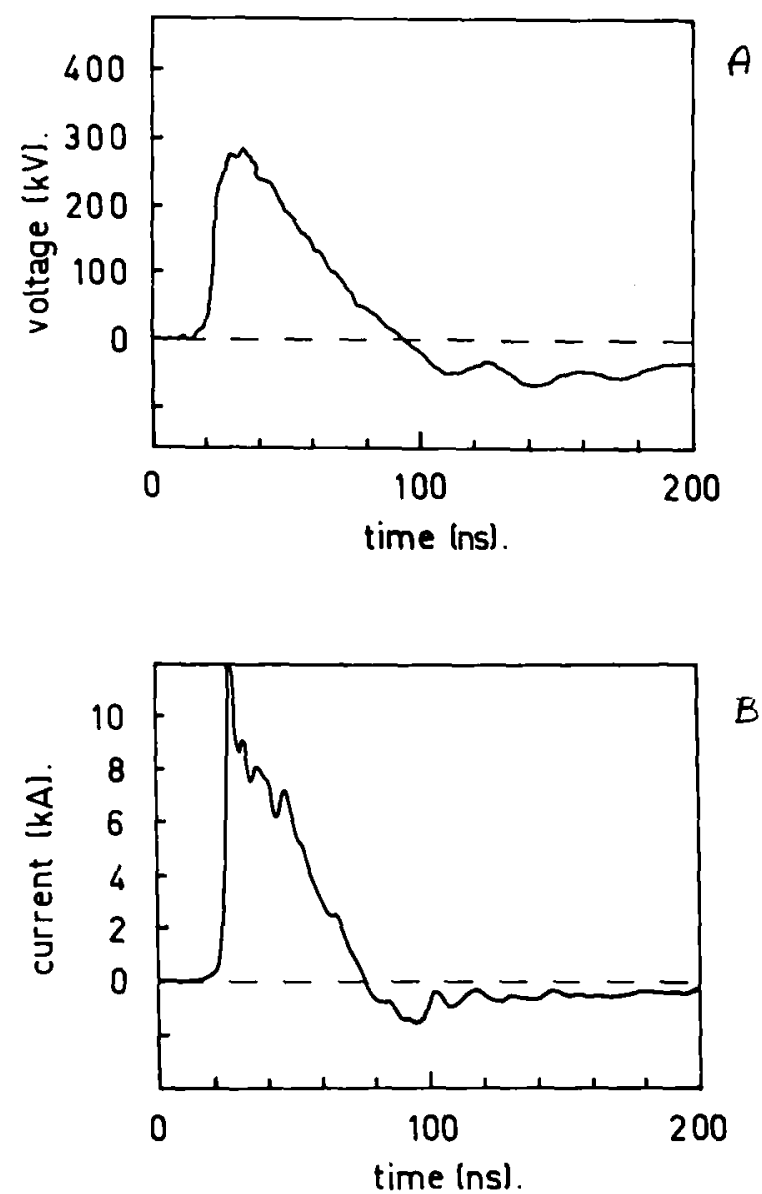

Fig. 10. (a) Voltage pulse and (b) current pulse at the vacuum diode as a function of time. The charging voltage is $60 \mathrm{kV}$ per stage.

the current pulse-width is about 50 nanoseconds and that the current increases with the charging voltage. The initial current peak is related to the charging of the diode capacitance, which is estimated to be $25 \mathrm{pF}$. From photographic observations of phosphorescence of Torr Seal on a perspex tube we found uniform excitation along the laser tube. The chamber is ended with two $\mathrm{MgF}_{2}$ windows, to one of which a quartz roof edge prism is attached as a total reflector, while the other serves as the output coupler.

All experiments described below have been performed at a charging voltage of $280 \mathrm{kV}$ and current of $7.5 \mathrm{kA}$ in 30 nanosecond pulses. From this we estimate a maximum current density of approximately $375 \mathrm{~A} / \mathrm{cm}^{2}$ and an average current of about $250 \mathrm{~A} / \mathrm{cm}^{2}$. The maximum electron energy inside the laser tube is approximately $170 \mathrm{keV}$. 


\subsection{THE $\mathrm{Kr}-\mathrm{F}_{2}-\mathrm{Ne}$ MIXTURE AT HIGH PRESSURE}

Studies on different buffer gases in a $\mathrm{KrF}^{*}$ laser showed that the choice depends on the gas pressure [14]. It is found that the best results are obtained for mixtures containing $\mathrm{Ne}$ as buffer gas at pressures above 10 bar. With the described system a pulse energy of about 0.5 Joule is obtained in a mixture containing $10 \mathrm{mbar} \mathrm{F}_{2}, 500 \mathrm{mbar} \mathrm{Kr}$, and $12 \mathrm{bar} \mathrm{Ne}$. The maximum current density is $375 \mathrm{~A} / \mathrm{cm}^{2}$, which corresponds to an average current of about 250 $\mathrm{A} / \mathrm{cm}^{2}$. In fig. 11 we plotted the extracted energy as a function of the total pressure with $\mathrm{Ne}$ as buffer gas. In all experiments we used a constant $\mathrm{F}_{2}$ pressure of $10 \mathrm{mbar}$ which was observed to be the optimized concentration of $F_{2}$. Each curve described the measurements with constant $\mathrm{Kr}$ pressure and a constant $\mathrm{Ar}$ pressure of $490 \mathrm{mbar}$. This presence of $\mathrm{Ar}$ is due to the fact that the available $\mathrm{F}_{2}$ was diluted in Ar gas. The curves of fig. 11 show that the maximum of each curve increases with decreasing $\mathrm{Kr}$ concentration and reaches a maximum for 500 mbar. The higher maximum is obtained for higher total pressure. We also

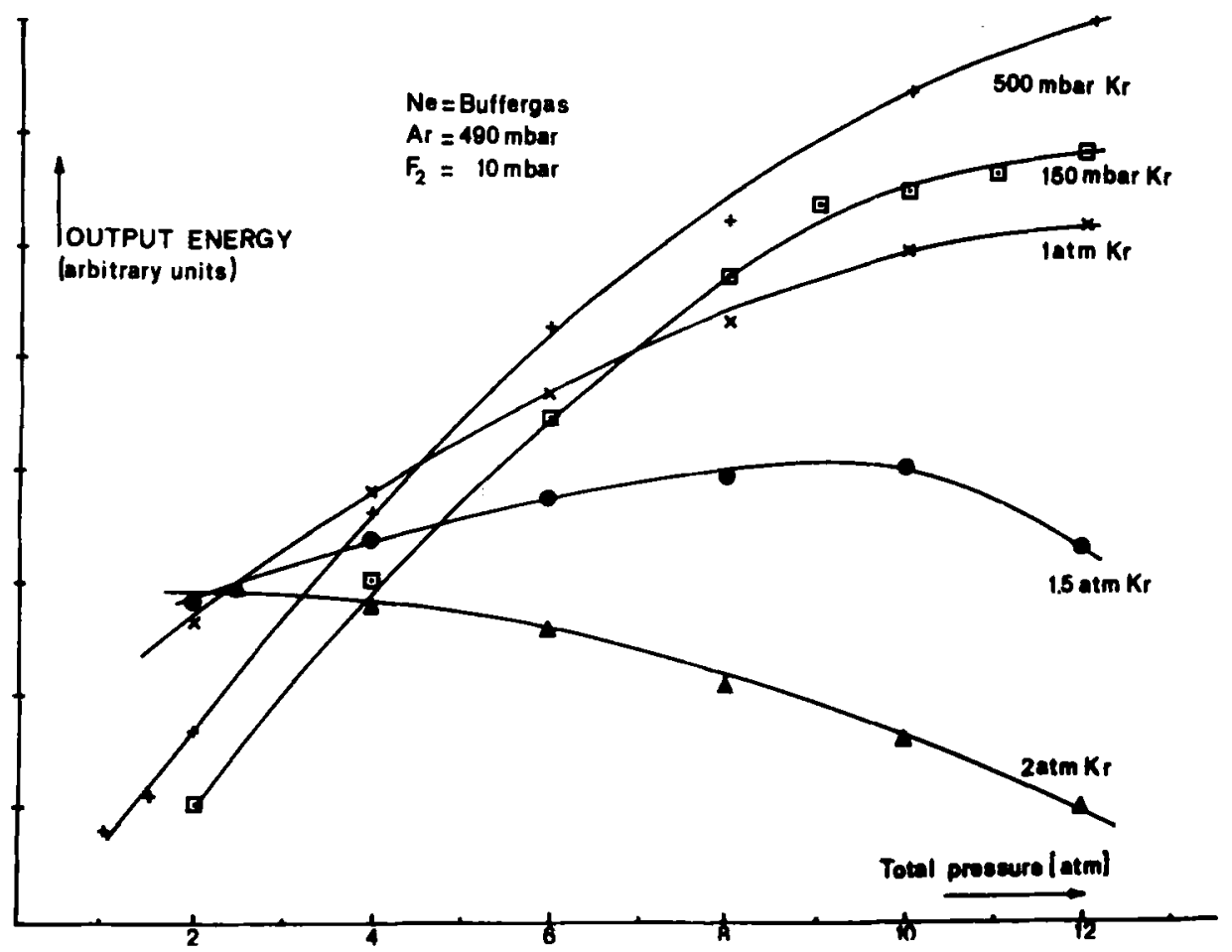

Fig. 11. The ouput energy of the four component mixture Ar-Kr-Ne-F as a function of the Ne pressure. The $\mathrm{Kr}$ pressure is used as parameter. The Ar gas is not necessary for the high performance. Its presence is due to the fact that the available $F_{2}$ was diluted in Ar. 
observed that if one chooses for a maximum total pressure below 4.5 bar a higher $\mathrm{Kr}$ pressure is more favourable. In this pressure range below 4.5 bar the use of $\mathrm{Ar}$ as buffer gas is even more favourable. The maximum admissible pressure of our system is 12 bar. We may extrapolate the results and conclude that the optimum $\mathrm{Ne}$ pressure will be about 15 bar.

\section{References}

[1] G.J. Ernst and W.J. Witteman, IEEE J. of Quantum Electronics, QE-7 (1971) 484.

[2] P.J.M. Peters, W.J. Witteman and Z. Krzyzanowski, Opt. Comm. 45 (1983) 193.

[3] A.L.S. Smith and S. Moffat, Opt. Comm. 30 (1979) 213.

[4] M.C. Skolnick, IEEE J. Quantum Electr., QE-6 (1970) 139.

[5] K.M. Abramski, J. van Spijker and W.J. Witteman, Appl. Phys. B 36 (1985) 149.

[6] V.J. Stefanov, J. Phys. E.: Sci. Instrum. 3 (1970) 1027.

[7] H. Jacobs, A.J. Harecman and J. Schumacher, J. Appl. Phys. 38 (1967) 3412.

[8] G.J. Ernst, Rev. Sci. Instrum. 48 (1977) 1281.

[9] D.J. Brink and V. Hasson, J. Appl. Phys. 49 (1978) 2250.

[10] G.J. Ernst and A.G. Boer, Opt. Comm. 44 (1982) 125.

[11] T.Y. Chang, Rev. Sci. Instrum. 44 (1973) 405.

[12] D.J. Bradley, D.R. Hull, M.H.R. Hutchinson and M.W. McGeoch, Opt. Comm. 14 (1975) 1.

[13] B.M.H.H. Kleikamp and W.J. Witteman, Opt. Comm. 49 (1984) 345.

[14] B.M.H.H. Kleikamp, P.J.M. Peters and W.J. Witteman, IEEE J. of Quantum Electronics QE-22 (1986) 219. 